\title{
Improved Sensitivity of Terahertz Label Free Bio-Sensing Application Through Trapped-Mode Resonances in Planar Resonators
}

\author{
Mihai D. Rotaru and Jan K. Sykulski, Fellow, IEEE \\ School of Electronics and Computer Science, University of Southampton, Highfiled, Southampton, SO17 1BJ, UK
}

\begin{abstract}
A design is proposed and investigated exploiting full wave numerical simulation of a dual resonant structure with an aim to sense small amounts of chemical and biochemical materials. The structure is energized with free space radiation in the terahertz regime. Thanks to its asymmetric geometry high quality trapped mode resonances are excited. By selectively loading the structure with only small amounts of probe material, a relatively large shift in the frequency response may be achieved. The concept is demonstrated through simulation, while optimization of the structure and the analyte loading is attempted.
\end{abstract}

Index Terms-Biomedical transducers, finite element methods, resonators.

\section{INTRODUCTION}

$\mathbf{R}$ ECENT technological advances, coupled with the ability to fabricate features on solid substrates with nanoscale precision, have fuelled the market of biochemical sensors, especially for DNA-based testing and sensing. Genetic diagnostics kits-DNA biosensors, gene chips and lab-on-chip DNA analyzers - have been developed. Methods using giant magnetoresistance effect biosensors with magnetic nanoparticle tags have been reported [1], [2], but most of the sensing techniques rely on detecting the hybridization of an unknown target to a known single stranded probe DNA molecule. Contemporary detection is based on fluorescent labelling, shown to be quite efficient, but having some drawbacks [3], thus creating a need for label-free detection schemes. Terahertz probing technology has the potential for label-free detection of the DNA binding state, as theoretical calculations predict several resonances in the $\mathrm{THz}$ range associated with interbackbone excitation of DNA molecules [4]. This possibility has recently been explored and a number of new approaches proposed [3], [5].

Conventional terahertz spectroscopy cannot be harnessed, mainly because of insufficient quantity of the analyte available for testing. High sensitivity terahertz sensors usually employ high quality $(\mathrm{Q})$ resonant structures whose frequency response is shifted by dielectric loading. Solutions based on microstrip resonators, evanescent field resonances and gratings have been proposed but lead to complications such as a need to integrate the $\mathrm{THz}$ source near the sensing element or complex coupling. Recent investigations have shown that high-Q resonances can be excited on to small asymmetric structures [6], [7]. These novel structures could be used as sensing elements for terahertz spectroscopy to determine the state of hybridization of small samples of DNA (femtomol level). To reduce the system complexity and lower the cost it may be useful to separate the reader from the sensor chip, as the chip can then be considered as an expendable part. In this paper we propose a simple but efficient structure that can sustain high-Q trapped mode resonances and also could be arranged in an array of resonators and integrated

Manuscript received May 04, 2010; accepted October 27, 2010. Date of current version April 22, 2011. Corresponding author: M. D. Rotaru (e-mail: mr@ecs.soton.ac.uk).

Color versions of one or more of the figures in this paper are available online at http://ieeexplore.ieee.org.

Digital Object Identifier 10.1109/TMAG.2010.2091946

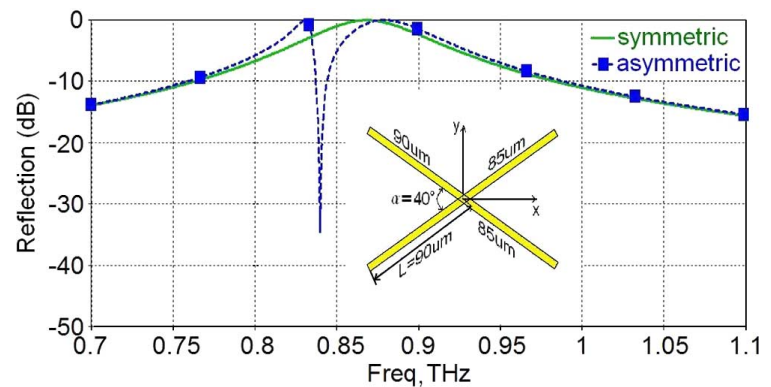

Fig. 1. Absolute values of the reflection coefficient of the $\mathrm{X}$ resonator for an infinite array configuration: symmetric and asymmetric (dotted line) cases.

into a chip that could be directly accessed from free space through terahertz radiation.

\section{RESONATOR CONFIGURATION}

A simple X-shaped resonator was simulated to obtain the reflection and transmission characteristics when illuminated with a plane electromagnetic wave in the terahertz regime. Initially a perfect electric conductor (PEC) structure with a thickness of $400 \mathrm{~nm}$ and width of $5 \mu \mathrm{m}$ was considered, hence metal losses were ignored. Fig. 1 depicts one cell from an array of such resonators. The total cell size was assumed to be $220 \times 220 \mu \mathrm{m}^{2}$. All reported numerical simulations have been performed using CST Microwave [8] at a unit cell level, with periodic boundary conditions in $x$ and $y$ directions, hence the array may be considered to be infinitely large. Floquet ports with two modes are introduced for the excitation of the structure on the top and bottom surface of the 3D cell. In this set-up a plane wave propagating normal to the $x y$ plane is modelled with mode 1 polarized in $y$ direction and mode 2 polarized in $x$ direction, respectively. The model is solved using the frequency domain solver on a tetrahedral mesh. The mesh is adapted first at $1.2 \mathrm{THz}$, the highest frequency of interest, and then a number of adaptively chosen frequency samples over the $0.7-1.2 \mathrm{THz}$ interval are solved using the same mesh. The reflection and transmission through the sample are then computed from the field solution.

For the symmetric X-shaped geometry, with all four arms equal, a single resonant frequency is observed in the frequency range $0.7-1.2 \mathrm{THz}$. The $\mathrm{X}$ resonator behaves like a moderate $\mathrm{Q}$ factor bandstop filter for waves polarized along its shorter dimension ( $y$-axis in this case), nevertheless the edges of the response are not sufficiently sharp to achieve high sensitivity when 
used as a sensing element. However, if asymmetry is introduced (by reducing the length of the two left arms), a dual resonance occurs, due to different parts of the $\mathrm{X}$ resonator becoming resonant at slightly different frequencies. This new resonance has a very sharp local maximum and a zero nearby (Fig. 1), which could be used as a sensing principle. Through dielectric loading of the structure it is expected that the sharp resonance observed will be shifted and therefore by comparing the frequency response of the structure loaded with a known sample with its response obtained for an unknown sample one can judge the nature of the unknown test analyte.

\section{TRAPPED Mode RESONANCE}

For a device such as the proposed resonator to be effective as a sensing element for small biochemical samples it requires high electric field concentration, as it is expected that the sample will interact with the E field changing its distribution and thus modifying the frequency response. The resonance quality factor-resonant frequency over the width of the resonance-could be used to quantify the ability of the structure to concentrate the electromagnetic field around itself. Most of resonant structures of this size do not have high quality factor resonances because they are strongly coupled to free space and therefore will suffer high radiation losses; moreover, due to their reduced size $(<$ wavelength), they are not able to provide a large enough confinement volume for the electromagnetic field to support high Q resonances. Recent studies [6], [7] have shown that high $\mathrm{Q}$ resonant modes could be excited in small structures if certain asymmetries were introduced in the resonant element. These high $\mathrm{Q}$ resonant modes are attributed to symmetric current modes ("trapped modes") and are not accessible in symmetric structures.

It is interesting to explore further the nature of the resonant response described in Section II. Our simulation results show a deep and narrow resonance at about $0.85 \mathrm{THz}$, for the size of the structure as in Fig. 1. This response occurs only for an incident wave with its electric field polarized in a direction orthogonal to the mirror line of the resonator, $y$-polarized in this case. For the polarization direction parallel to the mirror line, the $x$-polarized field, no such response was observed.

This very low reflection of the structure is due to two antisymmetric currents induced on the two unequal sides of the structure (Fig. 2(b)). At $0.85 \mathrm{THz}$ these currents are almost equal in amplitude and 180 degree out of phase. As a consequence the electric and magnetic dipole radiations from these currents will cancel each other; hence they will not interfere with the wave propagating through the structure. For frequencies immediately below and above $0.85 \mathrm{THz}(0.836 \mathrm{THz}$ and $0.863 \mathrm{THz}$ respectively) the induced current distribution is completely different (Fig. 2(a) and 2(c)) resulting in a dipole reflection dominating the response of the structure. At $0.836 \mathrm{THz}$, the induced current on the longer side of the structure dominates, whereas at 0.863 $\mathrm{THz}$ the current induced on the other shorter side dominates. The radiation from the structure interferes now with the electromagnetic wave that has induced the currents and the reflection at these two frequencies is high as shown in Fig. 1. It is useful to look at the distribution and the values of the electric field around the resonator structure. These plots (Fig. 2) reveal that the field is tightly distributed around the end legs for all three resonances
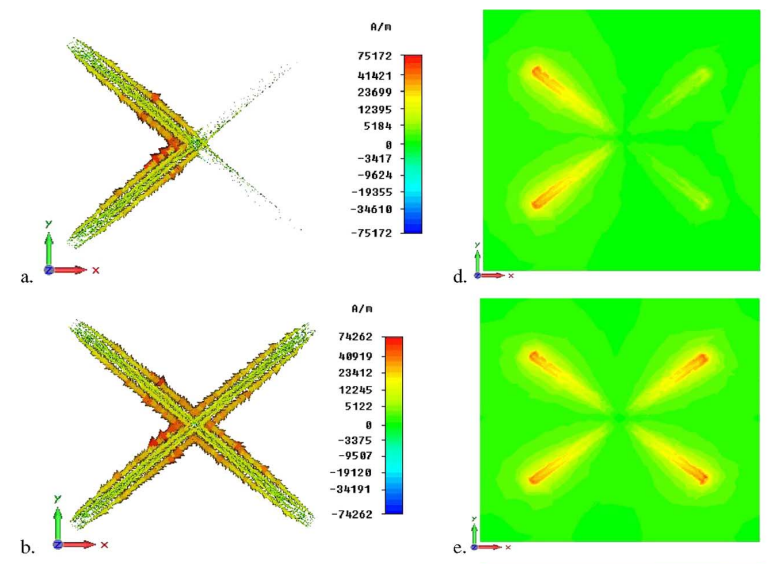

$\begin{array}{r}0 / \mathrm{m} \\ 1.54 \mathrm{ee} \\ 1.01 \mathrm{e} 7 \\ 7.08 \mathrm{e} 6 \\ 4.85 \mathrm{ee} 6 \\ 3.19 \mathrm{e} 6 \\ 1.97 \mathrm{e} 6 \\ 1.96 \mathrm{ee} \\ 3.88 \mathrm{e} 5 \\ 8\end{array}-$
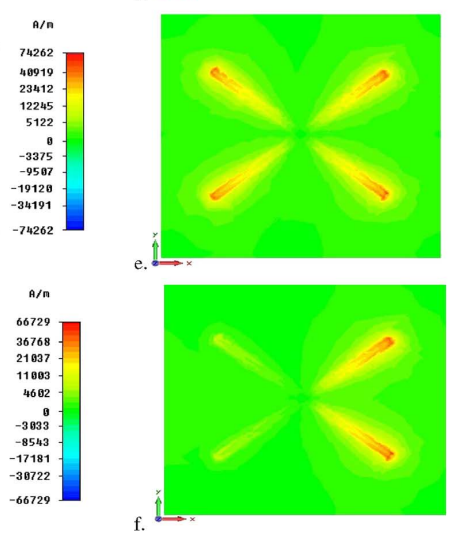

$0 / \mathrm{m}$
$1.60 \mathrm{e} 7$
$1.05 \mathrm{e} 7$
$7.38 \mathrm{e} 6$
$5.86 \mathrm{e6}$
$3.33 \mathrm{e} 6$
$2.85 \mathrm{eb}$
$1.11 \mathrm{e} 6$
4.9445
0
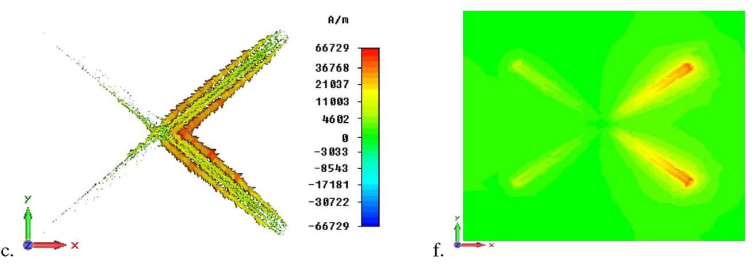

$0 / \mathrm{m}$

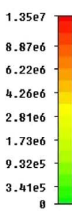

Fig. 2. Surface current distribution at $0.836 \mathrm{THz}$ (a), $0.85 \mathrm{THz}$ (b) and $0.863 \mathrm{THz}$ (c) and electric field distribution around the " $\mathrm{X}$ " resonator at $0.836 \mathrm{THz}$ (d), $0.85 \mathrm{THZ}$ (e) and $0.863 \mathrm{THz}$ (f).

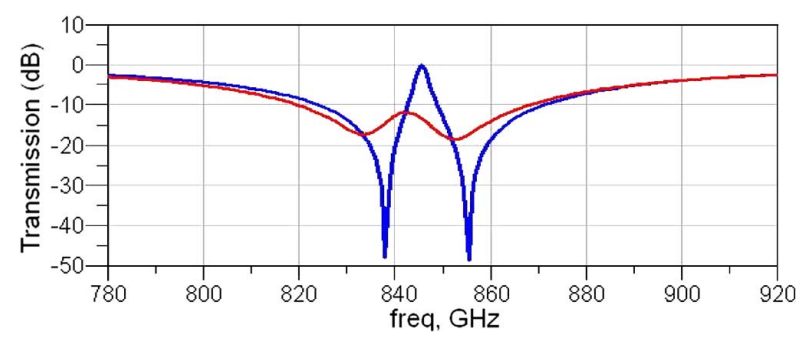

Fig. 3. Transmission through the "X" structure for PEC and gold.

described. Moreover, the intensity of the electric field is very high, especially at the extreme end of the X (Fig. 2).

At $\mathrm{THz}$ frequencies metal losses are substantial and have to be considered, unlike in the microwave region where they are negligible. To account for these losses all simulations were repeated with the PEC condition replaced by a lossy metal. The " $\mathrm{X}$ " structure was assumed to be made of gold, with the material properties found using the Drude model. The results indicate that the resonant behavior is unchanged; however, the quality factor is much reduced as expected. A comparison between the transmission spectra of lossless and lossy case is shown in Fig. 3. All three resonances are well preserved, but now the magnitude of the transmission of the trapped mode is well below $0 \mathrm{~dB}$, in fact the computed value is about $-12 \mathrm{~dB}$.

In order to quantify the ability of the resonator to confine the electromagnetic field, a further numerical study was undertaken. Two geometrical parameters, the angle alpha $(\alpha)$ between the two arms of the X shape and the length difference between the two sides of the structure, were considered. The quality factor $\mathrm{Q}$ of the resonator was calculated for a range of angles with the other parameter fixed; similarly Q was calculated for different lengths but with the angle fixed. The quality factor is calculated at the second resonance, dividing the frequency value at resonance by the bandwidth measured at $3 \mathrm{~dB}$ below the maximum in the transmission spectra. 

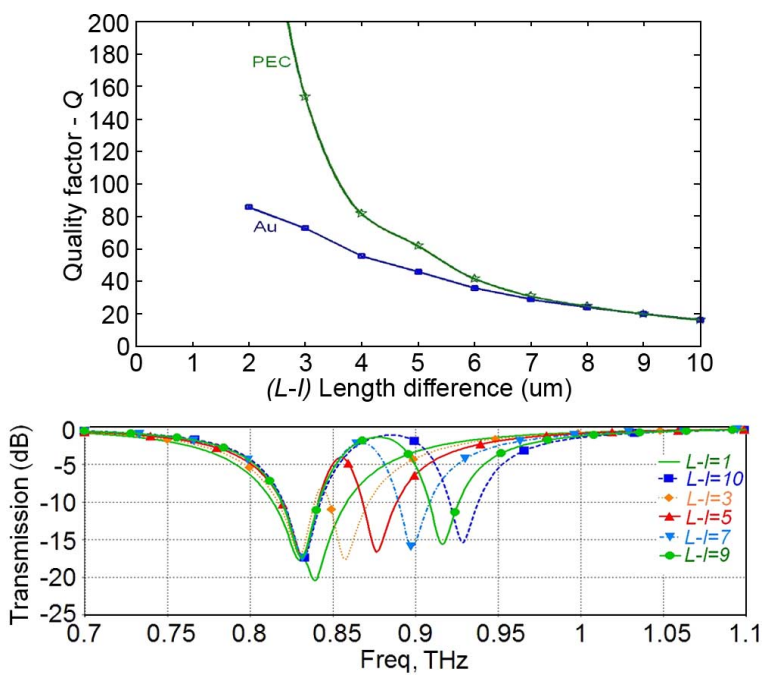

Fig. 4. Quality factor $\mathrm{Q}$ and transmission as a function of $L-l$.
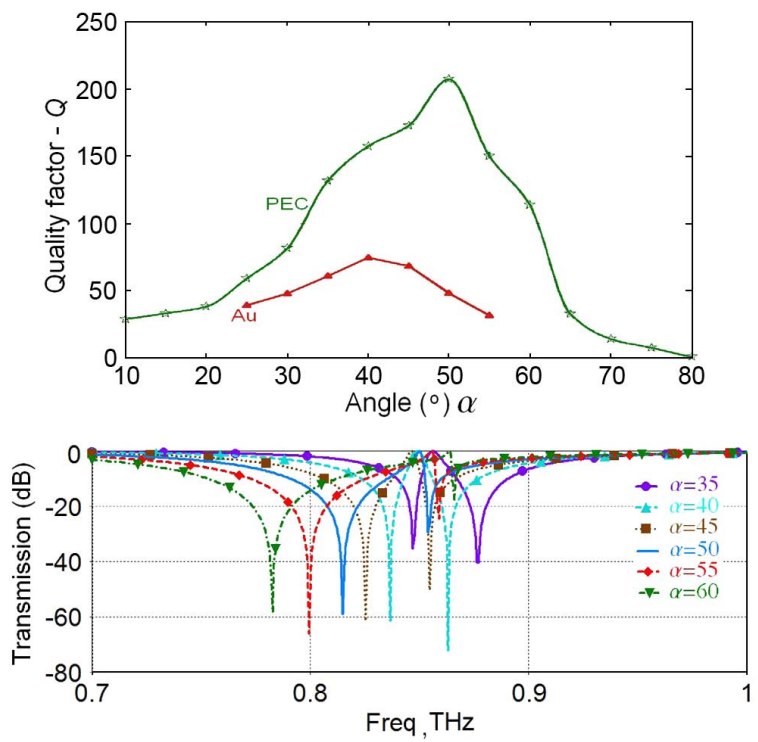

Fig. 5. Quality factor $\mathrm{Q}$ and transmission as a function of $\alpha$.

The results show that, for an " $\mathrm{X}$ " structure with a constant angle $\alpha=40^{\circ}$, when the difference between the lengths $(L-l)$ of the two sides increases, the second and third resonances shift towards higher frequency. The first resonance, due to the dipole radiation of the longer side of the structure, does not change, which was to be expected as $L$ is kept constant throughout. The quality factor, $\mathrm{Q}$, also varies, in the lossless case (PEC) monotonically decreasing with the increase in $(L-l)$ (Fig. 4). For the lossy case $\mathrm{Q}$ follows a similar pattern, but has a much smaller value and a maximum at $L-l=2 \mu \mathrm{m}$.

When $(L-l)$ is kept constant (at $3 \mu \mathrm{m}$ ) and the angle varied from $10^{\circ}$ to $80^{\circ}$, all three resonant frequencies are shifted but in a more complex fashion. First, as the angle increases from $10^{\circ}$ to $45^{\circ}$, all resonant frequencies are shifted towards lower values. Between $45^{\circ}$ and $80^{\circ}$, the first resonance keeps shifting downwards but the second and third resonances start moving towards higher values. The $\mathrm{Q}$ factor varies monotonically no more; instead it has a very distinct maximum at about $50^{\circ}$ for the lossless case and at $40^{\circ}$ when loss in the metallic structure is not ignored in the calculation (Fig. 5).

\section{SENSITIVITY ANALYSIS}

It is important to analyze the sensitivity of the proposed " $\mathrm{X}$ " structure for the sensing application. Therefore further numerical experiments were undertaken for loaded structures. The sensitivity was judged in terms of the amount of the frequency shift observed in the reflection response due to the load as compared with the unloaded case. An optimal structure based on previous results was chosen, with the highest quality factor, using gold as a metallic material. As shown in Figs. 4 and 5, the difference $L-l$ of $3 \mu \mathrm{m}$ and the angle of $40^{\circ}$ result in a high quality trapped mode resonance. Clearly high $\mathrm{Q}$ will yield enhanced sensitivity.

As explained in the introduction, the quantity of the analyte in biochemical applications is very small. A typical mono-molecular DNA film is about 10 to $50 \mathrm{~nm}$ thick with an area of about $750 \mu \mathrm{m}^{2}$ [3]. The electric properties considered here are in the range of 3.2 to 3.5 for $\epsilon_{\mathrm{r}}$ for a hybridized (double-stranded) DNA sample and $\epsilon_{\mathrm{r}}=1.9$ for denaturated (single-stranded) DNA [9]. The samples were considered to be lossless, which is a reasonable assumption for dry specimens.

The placement of the samples with respect to the sensing structure becomes critical when small sizes (especially the thickness) are involved. By inspecting the electric field plots one can evaluate where the maximum intensity field regions are and place the samples in those regions. From Fig. 2, it is clear that the maximum $\mathrm{E}$ field is around the legs of the " $\mathrm{X}$ " structure; however the position changes according to the resonance excited. When the trapped mode resonance is excited, all four legs seem to have similar high values of the E field around them. Two samples with the same properties and same sizes were used to load the sensor. The size considered was $35 \times 7 \mu \mathrm{m}^{2}$, while the thickness was varied from 100 $\mathrm{nm}$ to $10 \mathrm{~nm}$. This optimum size was found through a few extra simulations in which different size samples were tested. Due to a small thickness of the sample the mesh around and within the sample had to be carefully refined. Several layers of dummy objects were built around the sample to enforce the mesh to be fine enough in the area of interest ensuring reliable numerical results. Larger area coverage would have little effect on the sensitivity. The sample is positioned on top of the metal structure and it goes along the leg for $30 \mu \mathrm{m}$. It also extends out another $1 \mu \mathrm{m}$ on both sides, as well as $5 \mu \mathrm{m}$ on the top, hence the total area covered is $35 \times 7 \mu \mathrm{m}^{2}$. There are four possible combinations in which the two samples could be loaded on the "X" structure as shown in Fig. 6.

It is interesting to note that the thickness of the sample has a stronger effect on the sensitivity than the area. For example, for a $100 \mathrm{~nm}$ thick sample a clear difference-a shift of about $1.6 \mathrm{GHz}$ - can be seen between samples with the area of $35 \times$ $25 \mu \mathrm{m}^{2}$ and $35 \times 7 \mu \mathrm{m}^{2}$, whereas for $50 \mathrm{~nm}$ thick samples no difference is observed (Fig. 7). Moreover, a larger frequency shift is obtained for a thicker sample, e.g. a $5.25 \mathrm{GHz}$ shift for a $100 \mathrm{~nm}$ thickness, whereas only $3 \mathrm{GHz}$ for a $50 \mathrm{~nm}$ thickness.

The loading positions 3 and 4 (Fig. 6) have slightly better sensitivity compared to the other possible combinations ( $1 \& 2$, $1 \& 3$ and $1 \& 4)$. The $100 \mathrm{nmn}$ thick sample yields a shift of about $6.8 \mathrm{Ghz}$, while the $50 \mathrm{~nm}$ thick sample shows a $3.4 \mathrm{GHz}$ shift (Fig. 8). The main difference between the two loading conditions ( 1 and 2 against 3 and 4 ) is the fact that different resonances are influenced by the dielectric loads. For the loaded positions 1 


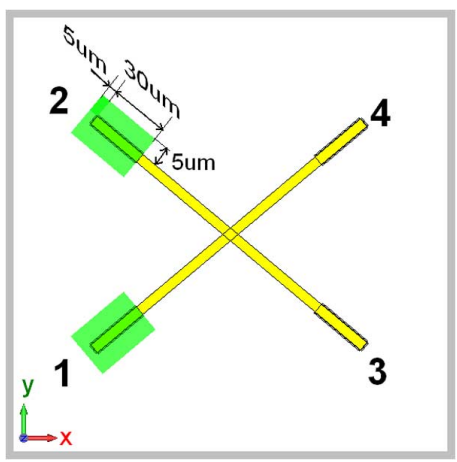

Fig. 6. Four possible loading sites on the "X" structure. The picture shows a loading for 1 and 2 with two $35 \times 15 \mu \mathrm{m}$ samples.
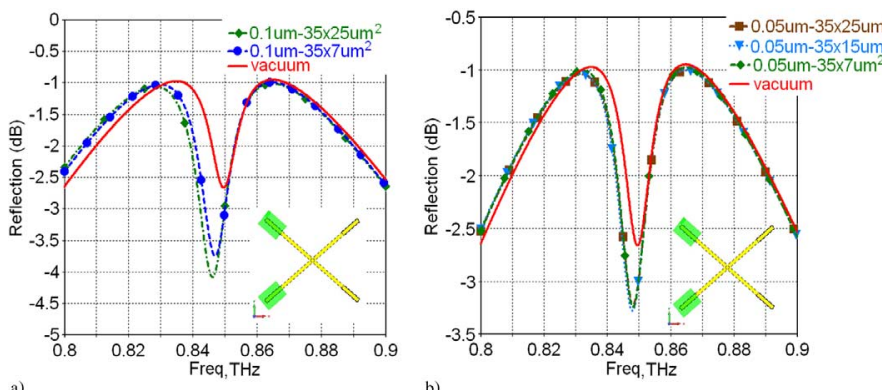

Fig. 7. Frequency shift upon dielectric loading $\left(\epsilon_{\mathrm{r}}=3.2\right)$ for positions 1 and 2. (a) $100 \mathrm{~nm}$ thick sample, (b) $50 \mathrm{~nm}$ thick sample.

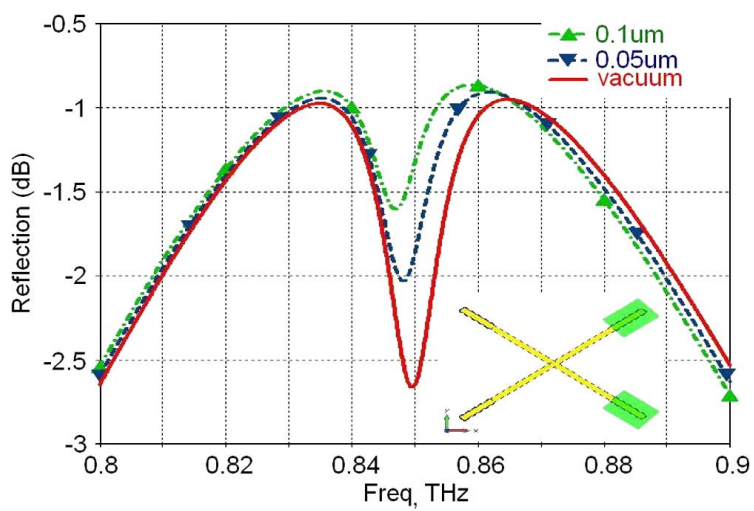

Fig. 8. Frequency shift upon dielectric loading $\left(\epsilon_{\mathrm{r}}=3.2\right)$ for positions 3 and 4; $100 \mathrm{~nm}$ and $50 \mathrm{nnm}$ thick samples.

and 2 the first and the trapped resonance frequencies are shifted downwards, but the third resonance frequency is unchanged. On the other hand, when 3 and 4 are used as loaded positions, the first resonant frequency does not move, while the other two are shifted downwards. This difference in behavior is also visible in the magnitude of the reflection for the trapped mode resonance when compared with the unloaded situation: in the former less of the power of the incoming wave is reflected back, whereas in the latter more is reflected back (Figs. 7 and 8).

Finally, a comparison is made in Fig. 9 between a loaded structure with a material having $\epsilon_{\mathrm{r}}=1.9$-corresponding to a denaturated (single-stranded) DNA-and a loaded resonator

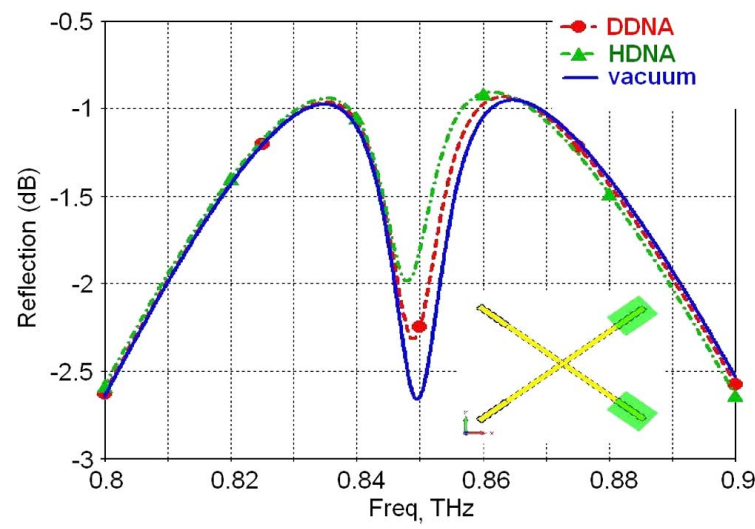

Fig. 9. Frequency shift upon dielectric loading, with $\epsilon_{\mathrm{r}}=3.5$ and $\epsilon_{\mathrm{r}}=1.9$ respectively, for the position 3 and 4 and a $50 \mathrm{nnm}$ thick sample.

with a hybridized (double stranded) DNA that has $\epsilon_{\mathrm{r}}=3.5$ [9]. The different response of the resonator for the two types of loading may be clearly seen. The relative frequency shift between the DDNA and HDNA is about $2.5 \mathrm{GHz}$.

\section{CONCLUSIONS}

In this work we have proposed and numerically analyzed a simple but efficient structure that could be used for high sensitivity terahertz chemical and biochemical sensors. The concept is based on the excitation of high quality "trapped modes" within the element cells of a frequency selective surface or a metafilm. The existence of trapped modes is paramount to achieve high sensitivity of such a scheme. Hence electromagnetic design of such structures harnessing numerical field simulation is very important. The analysis has shown that the shift in the frequency response upon loading with very small samples can be substantial as long as the samples are positioned in high electric field regions.

\section{REFERENCES}

[1] S. X. Wang and G. Li, "Advances in giant magnetoresistance biosensors with magnetic nanoparticle tags: Review and outlook," IEEE Trans. Magn., vol. 44, no. 7, 2008.

[2] L. Xu, H. Yu, S.-J. Han, S. Osterfeld, R. L. White, N. Pourmand, and S. $\mathrm{X}$. Wang, "Giant magnetoresistive sensors for DNA microarray," IEEE Trans. Magn., vol. 44, no. 11, 2008.

[3] C. Debus and P. H. Bolivar, "Frequency selective surface for high sensitivity terahertz sensing," Appl. Phys. Lett., vol. 91, 2007.

[4] L. L. Van Zandt and V. K. Saxena, "Millimeter-microwave spectrum of DNA: Six prediction for spectroscopy," Phys. Rev. A, T, vol. 39, no. 5, pp. 2672-2674, Mar. 1989.

[5] M. Nagel, M. Forst, and H. Kurtz, "THz biosensing devices: Fundamentals and technology," J. Phys.: Condens. Matter, vol. 18, pp. S601-S617, 2006.

[6] S. Prosvirnin and S. Zouhdi, Advances in Electromagnetics of Complex Media and Metamaterials. New York: Kluwer, 2003, pp. 281-290.

[7] V. A. Feodotov, M. Rose, S. L. Prosvirnin, N. Papasimakis, and N. I. Zheludev, "Sharp trapped-mode resonances in planar metamaterials with broken structural symmetry," Phys. Rev. Lett., vol. 99, no. 14, pp. 147401-1-147401-4, 2007.

[8] CST Microwave Studio [Online]. Available: www.cst.com

[9] T. Baras, T. Kleine-Ostmann, and M. Koch, "On-chip THz detection of biomaterials: A numerical study,” J. Biol. Phys., vol. 29, pp. 187-194, 2003. 